\title{
Time Exceedances for High Intensity Solar Proton Fluxes
}

\author{
Michael A. Xapsos, Senior Member, IEEE, Craig A. Stauffer, and Thomas M. Jordan, Member, IEEE
}

\begin{abstract}
A new model is presented for the total time during a space mission that specified solar proton flux levels are exceeded. It is applicable to any time period during the solar cycle and covers a broad proton energy range for both shielded and unshielded conditions.
\end{abstract}

Index Terms—-solar particle event, time exceedance, worst case
flux

\section{INTRODUCTION}

Developing and implementing strategies to deal with the space radiation environment is critical for new robotic and manned exploration initiatives. In order to have reliable and cost-effective spacecraft design and implement new space technologies accurate models that view things from varying perspectives are needed for estimating radiation risks. Underestimating radiation levels leads to excessive risk and can result in degraded system performance and loss of mission lifetime. Overestimating radiation levels can lead to use of excessive shielding, reduced payloads, over-design and increased cost.

Evaluating the risk due to solar particle events is a significant concern for all space missions, especially those away from the protective shielding effects of the Earth's magnetic field. Due to the difficulty in forecasting the occurrence and magnitude of solar particle events [1] probabilistic approaches are widely used to characterize events. In this regard, useful models have been developed to describe cumulative fluences [2-6] and worst case events $[7,8]$ at a given level of confidence over the course of a mission. For the situation of worst case events it is also common to pick a severe event such as the well known one that occurred in October 1989 and assume this is worst case for the mission, as is done in the CREME96 suite of codes [9].

For the situation of worst case events additional information can be required by the space system designer. Besides knowing a worst case flux over a period of time, i.e., the worst 5 minutes, worst day and worst week given in CREME96, it is also useful for the designer to know how much total time

Manuscript received April 29, 2011. This work was supported in part by the NASA Goddard Space Flight Center Internal Research and Development Program.

M. A. Xapsos is with NASA Goddard Space Flight Center, Greenbelt, MD 20771 USA (e-mail: Michael.A.Xapsos@nasa.gov).

C. A. Stauffer is with MEI Technologies, Seabrook, MD 20706 USA.

T. M. Jordan is with EMPC, Gaithersburg, MD 20885 USA. during the mission that a pre-determined flux level is exceeded. The reason is this allows a straight forward assessment of the mission time period during which there should be reliable system or instrument operation. In many instances this is closely related to the design goals of the mission. For example, the goal of the NASA Solar Dynamics Observatory (SDO) instrumentation is to capture essentially complete data over 22 72-day periods during its 5 years of operations, allowing for data loss due to planned and unplanned events, the latter of which includes radiation. Another example is the Space Environment Tesbed (SET) payload on the AFRL Demonstration and Science Experiment (DSX) spacecraft, that has an established success criterion of delivering $95 \%$ of the data for 40 weeks out of the 1 year of planned operations. This work develops a model for solar proton fluxes that contributes directly to these type of spacecraft design assessments.

The model developed in this paper evaluates the expected total time during a mission that a specified solar proton flux level is exceeded. This can be viewed as the mission time period during which an instrument, for example, may not operate reliably. The corresponding flux-energy spectrum bounding this period of unreliability is then constructed. This is done for both the unshielded case and for shielding levels that are appropriate for modern spacecraft. This complements the currently available models for solar protons that are used for spacecraft design.

\section{METHODS}

\section{A. Data Base}

The solar proton flux data used for this study have been described previously [10]. The data base consists of 28 years of flux-time measurements spanning the period from 1973 to 2001, during which the Goddard Medium Energy (GME) instrument on the Interplanetary Monitoring Platform-8 (IMP8) instrument operated nearly continuously. This is approximately 18 years during solar maximum and 10 years during solar minimum periods. The IMP-8 spacecraft orbit is a near circular one at approximately 35 Earth radii and is therefore well positioned to measure interplanetary particle fluxes. The GME data has a proton energy range of 0.88 to $485 \mathrm{MeV}$. These data have been supplemented with data obtained from the Geostationary Operational Environmental 
Satellites (GOES) beginning in 1986. The reason is that the GME instrumentation saturates during very high flux levels while the GOES instrumentation performs better. Reference 10 gives the details of how these data sets are combined so the best features of each can be taken advantage of. We are currently working on updating the data from 2002 to the present. Since this time period must be based almost entirely on the GOES data, we are proceeding cautiously to make sure the entire data set is consistent. This update is expected to be included in the results for the final paper.

\section{B. Model Calculations}

This model is based on a relatively straight forward, although computationally intensive, direct analysis of the solar proton flux time series (flux vs. time measurements) in our data base. The direct analysis of the time series avoids some of the usual difficulties that are encountered when constructing solar particle event models. One such difficulty is the unavoidably arbitrary definition of what constitutes an event. This can be particularly difficult when there are several rises and falls in the flux values before the flux returns to the background rate. Once events are defined it is commonly assumed that they are independent of one another, although this may not be the case [1].

The above difficulties are avoided in the current model. The approach is to choose a time period during the solar cycle corresponding to the mission of interest and calculate the amount of time during this period that a pre-determined flux level is exceeded. The flux level is then incremented and the calculation repeated. This is continued until the maximum observed flux level for that time period is reached. Time periods are referenced to the peak period of the solar cycle as determined by the maximum sunspot number. This is taken as 1968.9, 1979.9, 1989.9 and 2000.2 for the last 4 cycles. The above procedure is repeated for the full range of energy in the flux data time series. The integral energy spectra of the data base range from $>0.88$ to $>327 \mathrm{MeV}$.

These results are most relevant for mission planners if they are tied to shielding levels. For the purpose of the shielding calculations, we considered times when flux levels were above background. This totals approximately 10,800 energy spectra of 30 minute duration in the current data base. Transport calculations for each of these proton energy spectra was done using the NOVICE code [11] for a solid aluminum sphere geometry. Calculations of the amount of time various flux levels are exceeded during the period of interest are then repeated for different shielding levels.

\section{RESULTS}

Although the model can be implemented for any period of time during the solar cycle, for the purpose of this summary we will restrict ourselves to the solar maximum time period since this is generally when the highest solar proton flux levels are observed. Calculations were done as described in section IIB. The results presented here are for the entire 18 years of solar maximum data in our data base. Figure 1 is a plot of the number of hours per solar maximum year that the proton flux exceeds the value shown on the x-axis in units of protons per square centimeter per second per steradian. This is shown for various energy ranges. For example if one is interested in the $>11.1 \mathrm{MeV}$ energy group it is seen that a flux level of $10^{4}$ $\mathrm{p} /\left(\mathrm{cm}^{2}\right.$-s-sr $)$ is exceeded about 1 hour per year on average while a flux level of $10^{3}$ is exceeded about 1 day per year on average during solar maximum. The continuous range of information available in this analysis is much more complete than what is obtained from analysis of a single worst case event. The flux range shown in the figure extends from the maximum observed flux in the data base to 2 orders of magnitude down from that value for each energy group. The different perspective of using "time exceedances" also lends itself to analysis of reliable periods of operation of spacecraft instrumentation.

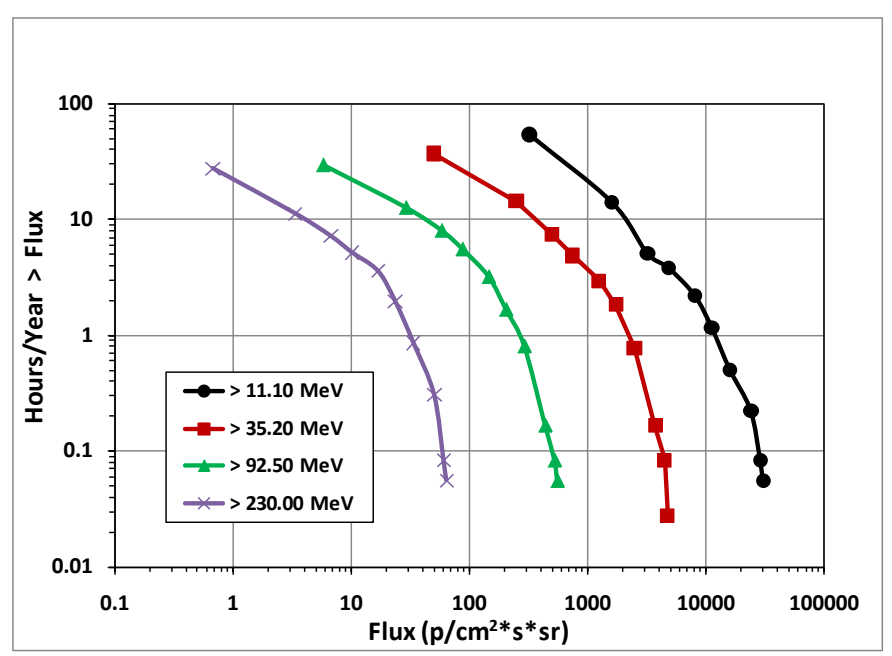

Fig. 1. Number of hours per year during solar maximum that the proton flux is expected to exceed the value shown on the abscissa for 4 energy groups: > 11.1, > 35.2, > 92.5 and $>230$ $\mathrm{MeV}$. Results are for unshielded fluxes.

The results shown in figure 1 are for unshielded fluxes. As mentioned before these results would be more useful if they could be determined for different levels of shielding. Thus, shielding calculations were done for each 30 minute long energy spectrum in the data base over the 18 year period of interest that fluxes were above background. The result is a time series of fluxes that spans 18 years for each level of shielding considered. Example model results including shielding are shown in figure 2 for the $>35.2 \mathrm{MeV}$ energy group. It is seen that the amount of time the flux level is above a threshold value is significantly influenced by shielding for this case. For example, it is expected to be above a flux level of $10^{3} \mathrm{p} /\left(\mathrm{cm}^{2}-\mathrm{s}-\mathrm{sr}\right)$ for 4 hours per year without shielding and less than 1 hour per year with 300 mils of $\mathrm{Al}$ shielding. Differences between the unshielded and shielded cases are more propounced for low energy and less pronounced for the high energy cases. 


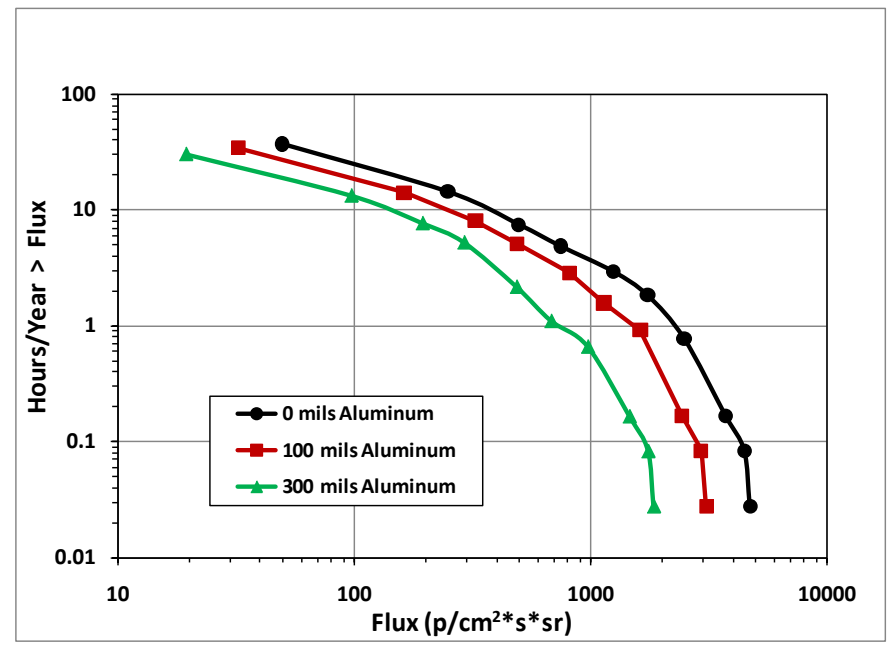

Fig.2. Number of hours per year during solar maximum that the proton flux is expected to exceed the value shown on the abscissa for $>35.2 \mathrm{MeV}$ protons with 0, 100 and 300 mils of aluminum shielding in a solid sphere geometry.

Since this approach of calculating time exceedances can generate a lot of results it is of interest to look for a more compact way to view them. One possibility is to calculate a flux vs. energy spectrum for a given time exceedance level. As an example, suppose a mission can afford to lose only one hour of data per solar maximum year due to high incidences of solar proton fluxes. A proton flux vs. energy plot for the one hour per year exceedance can be constructed by using results such as those shown in figure 1 . The various flux levels corresponding to a y-axis value of 1 hour per year are determined for each energy group. A plot of flux vs. energy can then be made as shown in figure 3 for the unshielded case. Each point on this plot represents the flux for the corresponding energy group that will be exceeded one hour per year on average. This 1-hour per year exceedance time holds only for individual points on the curve. It is not the case that the entire spectrum as a whole is exceeded 1 hour per year. Results for the shielded cases can be obtained in a similar fashion. A simpler way to obtain the shielded results, which has been verified, is to perform the transport calculation on the unshielded spectrum. These results are illustrated in figure 3, which is a particularly attractive representation because of its compactness as well as its close resemblance to measured solar proton event spectra.

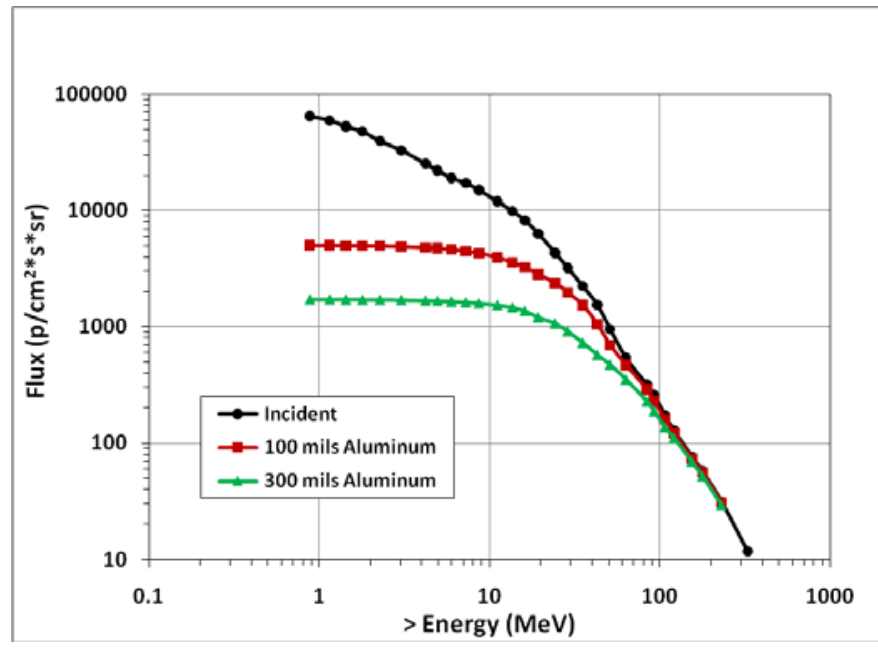

Fig.3. Flux vs. proton energy spectra for a 1-hour per year time exceedance. See text for interpretation. Results are shown for 0, 100 and 300 mils of aluminum shielding.

\section{SUMMARY AND CONCLUSIONS}

This paper has presented a model of high intensity solar proton fluxes that is complementary to existing models. Results show the amount of time during a mission that high intensity solar proton flux levels are expected to be exceeded. This has been done for a broad range of proton energies and for shielding thickness relevant for spacecraft application. The continuous spectrum of flux levels considered provides more complete information than worst case models based on a single event. This approach of using "time exceedances" as the relevant parameter is a natural way to tie results into unreliable periods of spacecraft or instrument operation, which is a key element of scientific data budgets.

In the full length paper the data base will be updated to include solar proton data from 2002 to the present, although there have been few events for the last 5 years. More results will be presented, including comparisons with the solar minimum time period.

\section{REFERENCES}

[1] M. A. Xapsos, C. Stauffer, J.L. Barth and E.A. Burke, "Solar Particle Events and Self-Organized Criticality: Are Deterministic Predictions of Events Possible?”, IEEE Trans. Nucl. Sci., vol. 53, no. 4, pg. 18391843, Aug. 2006.

[2] J.H. King, "Solar Proton Fluences for 1977-1983 Space Missions", J. Spacecraft, Vol. 11, 401-408 (1974).

[3] J. Feynman, G. Spitale, J. Wang and S. Gabriel, "Interplanetary Fluence Model: JPL 1991”, J. Geophys. Res., Vol. 98, 13281-13294 (1993).

[4] M.A. Xapsos, G.P. Summers, J.L. Barth, E.G. Stassinopoulos and E.A. Burke, "Probability Model for Cumulative Solar Proton Event Fluences”, IEEE Trans. Nucl. Sci., Vol. 47, No. 3, 486-490 (June 2000).

[5] R.A. Nymmik, "Probabilistic Model for Fluences and Peak Fluxes of Solar Energetic Particles”, Radiation Mesurements, Vol.30, 287-296 (1999).

[6] M. A. Xapsos, C. Stauffer, T. Jordan, J.L. Barth and R.A. Mewaldt, "Model for Cumulative Solar Heavy Ion Energy and Linear Energy Transfer Spectra”, IEEE Trans. Nucl. Sci., vol. 54, pg. 1985-1989, Dec. 2007. 
[7] M.A. Xapsos, G.P. Summers and E.A. Burke, "Probability Model for Peak Fluxes of Solar Proton Events”, IEEE Trans. Nucl. Sci., Vol. 45, 2948-2953 (1998).

[8] M.A. Xapsos, G. P. Summers, J.L. Barth, E.G. Stassinopoulos and E.A. Burke, "Probability Model for Worst Case Solar Proton Event Fluences”, IEEE Trans. Nucl. Sci., Vol. 46, 1481-1485 (1999).

[9] A.J. Tylka et al., "CREME96: A Revision of the Cosmic Ray Effects on Microelectronics Code”, IEEE Trans. Nucl. Sci., Vol. 44, 2150-2160 (1997).

[10] M.A. Xapsos, C. Stauffer, G.B. Gee, J.L. Barth, E.G. Stassinopoulos and R.E. McGuire, "Model for Solar Proton Risk Assessment", IEEE Trans. Nucl. Sci., Vol. 51, 3394-3398 (2004).

[11] T. M. Jordan, "An adjoint charged particle transport method," IEEE Trans. Nucl. Sci., vol. 23, no. 6, pp. 1857-1861, Dec. 1976. 